\title{
A TECHNIQUE FOR TRANSEPTAL LEFT HEART CATHETERIZATION VIA THE RIGHT EXTERNAL JUGULAR VEIN
}

\author{
BY \\ S. BEVEGÅRD, E. CARLENS, B. JONSSON, AND I. KARLÖF \\ From the Laboratory of Clinical Physiology, Thoracic Clinic, Karolinska Sjukhuset, Stockholm 60
}

With the aid of right heart catheterization information about the pressure in the left atrium can be obtained indirectly using the catheter in the pulmonary arterial wedge position. For an exact study of diseases within the left chambers of the heart it is important to measure the pressure in the left ventricle and to estimate the pressure gradients across the mitral valve and the left ventricular outflow tract.

Several methods for left heart catheterization have been used. Which method one should choose is dependent on the purpose of the examination. For physiological studies the following requirements should be fulfilled. (1) Catheterization should not cause a marked change of the heart function and it should be possible to define to what extent function has changed. (2) When the catheter has been introduced a steady circulatory state should be obtained before estimating the pressures and the cardiac output. (3) It should be possible to study the haemodynamics during exercise. (4) The procedure should not produce more morbidity than a right heart catheterization.

Transbronchial puncture of the left atrium, as described by Facquet, Lemoine, Alhomme, and Lefebvre (1952), Allison and Linden (1953), and Morrow, Braunwald, Haller, and Sharp (1957), has been widely used and very few complications have been reported. We have performed catheterization according to this method in 42 cases without serious complications, but bronchoscopy sometimes changes the haemodynamics considerably. Fourteen of our patients suffered from bronchial secretion and the arterial oxygen saturation was found to decrease. Posterior percutaneous puncture of the left atrium introduced by Björk, Malmström, and Uggla (1953) seems to us to be risky (Bagger, Björk, and Malmström, 1957). The technique of puncture of the left atrium from the suprasternal notch using a thin needle, described by Radner (1955), is limited to pressure recordings in the atrium.

Direct transthoracic puncture of the left ventricle has been used by Ponsdomenech and Beato Nuñez (1951) for injection of contrast medium in cardioangiography and by Brock, Milstein, and Ross (1956) for pressure recordings. In our hospital this method has been mainly used for cardioangiography under general anaesthesia (Kjellberg, Nordenström, Rudhe, Björk, and Malmström, 1960). Before the injection of contrast medium the pressures in the left ventricle and a systemic artery have always been recorded. Until now 130 patients have been examined in this way with no mortality. But puncture of the ventricular wall interferes with heart function. Arrhythmias occur and the systemic pressure may change. Therefore the method seems not to be suitable for haemodynamic studies and especially during exercise.

Catheterization of the left ventricle in a retrograde direction from the aorta (Zimmerman, Scott, and Becker, 1950) fulfils the requirements mentioned above but may be difficult in cases of aortic stenosis.

A technique for puncture of the atrial septum was described by Ross (1959). Through a catheter, introduced into the right atrium from the saphenous vein, a needle is advanced and the septum is punctured. A thin catheter is passed through the needle into the left atrium and further into the ventricle. This method has been used in 140 cases without serious complications (Morrow, Braunwald, and Ross, 1960). For studies during leg exercise, however, this method seems less suitable. We have used a technique of septal puncture passing the needle from the right external jugular vein. This method will be described and our clinical experience reported. According to Swan (1959), a similar method has been used by Donald in dogs. 


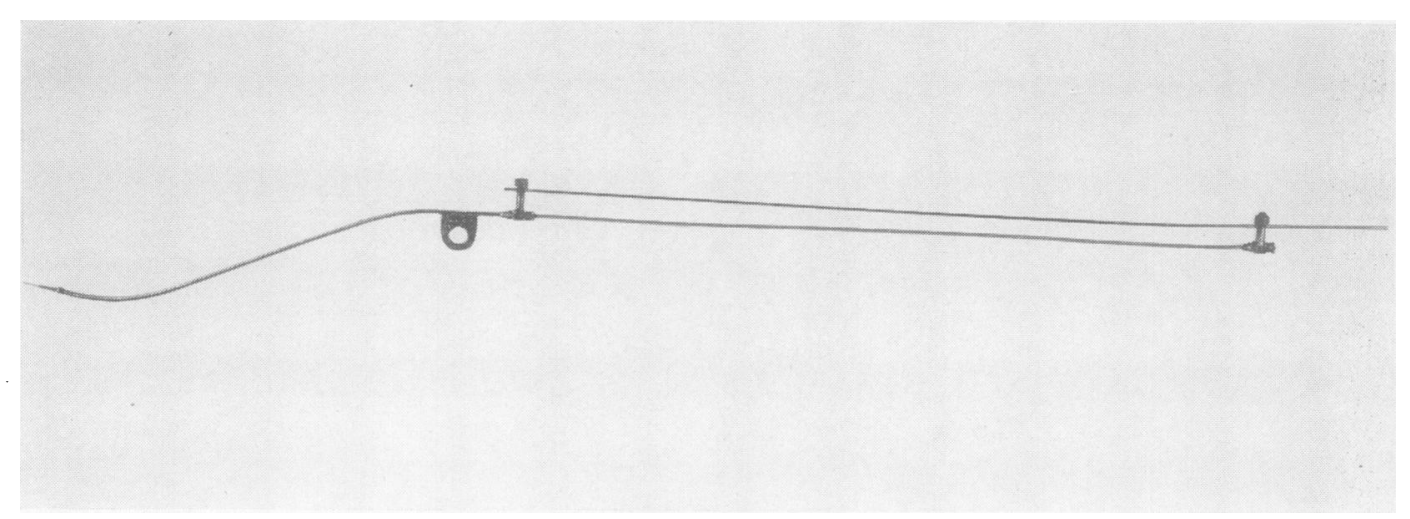

FIG. 1.-The instrument (for description see text).

\section{MeTHOD}

The instrument (Fig. 1) consists of a slightly S-shaped $25 \mathrm{~cm}$. long metal tube with an inner diameter of $2.5 \mathrm{~mm}$. and an outer diameter of $3 \mathrm{~mm}$. The lower end is somewhat olive-tipped serving as a rest or support against the atrial septum during the puncture. In its upper part the tube is provided with a wing-shaped handle. Into this tube is introduced a $70 \mathrm{~cm}$. needle (outer diameter $1.10 \mathrm{~mm} .=19$ gauge) over which a radio-opaque, $68 \mathrm{~cm}$. long teflon catheier (outer diameter $2.10 \mathrm{~mm}$.) is drawn. At its lower end the teflon catheter is adapted to the inner needle. The upper ends of the needle and the metal tube are each

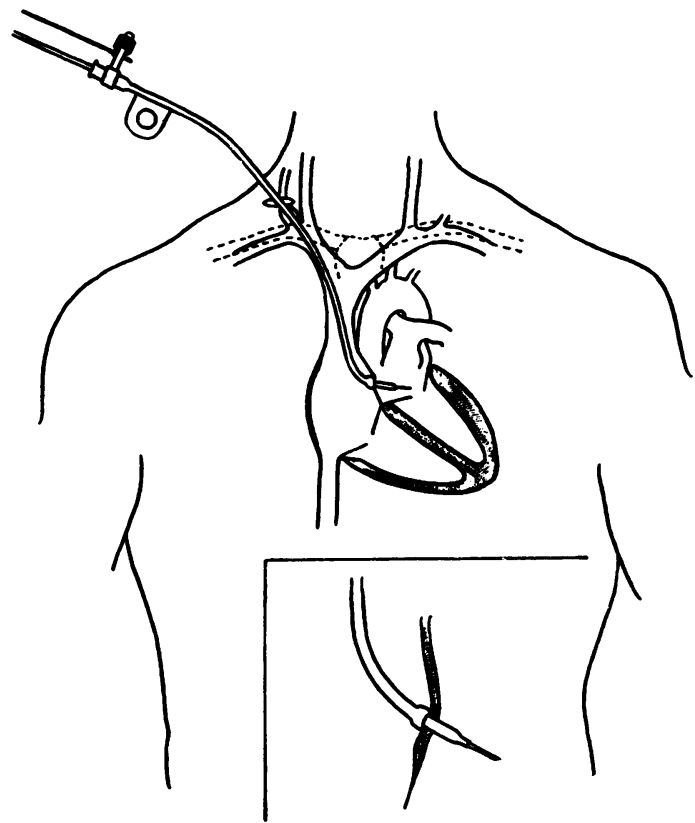

FIG. 2.-Schematic illustration of the position of the instrument during puncture of the atrial septum. provided with an attachment and can be fixed to each other with a graduated metal rod. With the help of the graduation of this metal rod the positions of the needle point and of the catheter point can be exactly determined in relation to the lower end of the metal tube, which is of course necessary if the attempt to perform a puncture is to be repeated. This graduated metal rod, which makes the method somewhat complicated, can be dispensed with if one can in a satisfactory way mark the teflon catheter at the outer end of the metal tube. This has hitherto not been possible, but will be feasible in the near future. The teflon catheter is fixed to the needle by means of a little handle with a clamping screw.

The right external jugular vein is exposed with a small incision 2-3 cm. above the clavicle. The

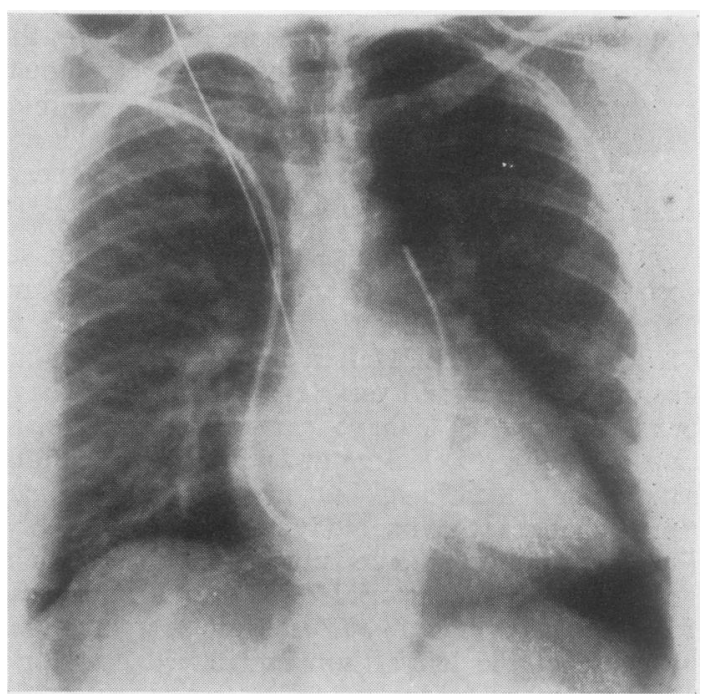

FIG. 3.-Radiograph showing catheters in the right and left heart. A double-lumen catheter is placed with the end-hole in the main pulmonary artery and the side-hole in the right ventricle. A tefion catheter, with steel wire inserted, is placed with the tip in the left ventricle. 
instrument is assembled and the upper end of the needle is attached by a plastic tube to a pressure receptor. With the needle in the withdrawn position the instrument is introduced into the vein and pushed down with fluoroscopic guidance to the right atrium. The instrument is then rotated in such a way that the olive-tipped end is resting with slight pressure against the atrial septum posteromedially. The position of the instrument during puncture is reproduced schematically in Fig. 2. While observing the pressure curve one presses the needle in about $2 \mathrm{~cm}$. When the left atrial pressure has been obtained, the needle is fixed to the metal tube in the way described above. The clamp-screw is loosened so as to free the catheter from the needle. The catheter is pushed over the needle into the left atrium and eventually further down into the left ventricle. The needle and the metal

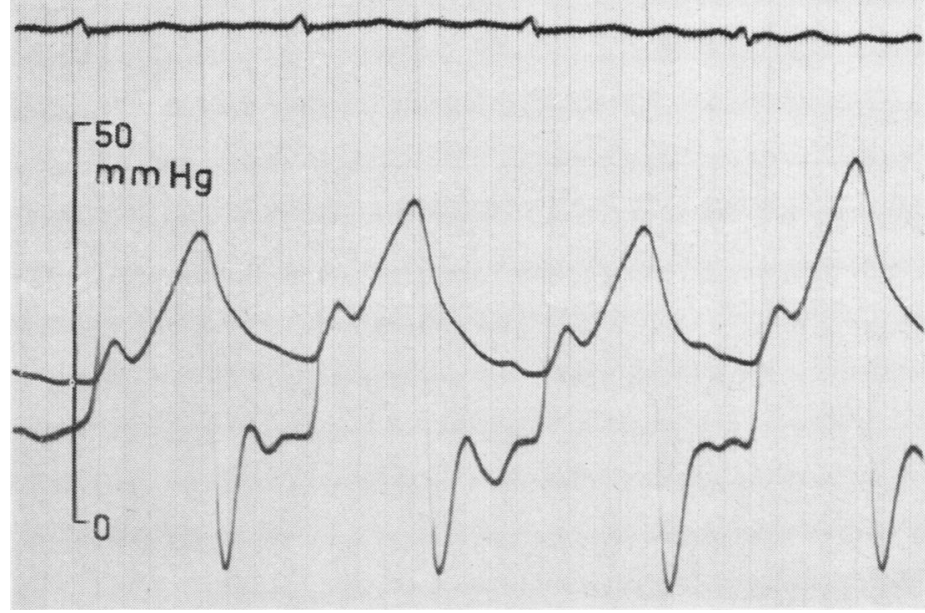

FIG. 4.-Simultaneous pressure recording from the left atrium and left ventricle 'in a case with combined mitral valvular lesion and aortic incompetence. One catheter was passed in a retrograde direction from the aorta to the left ventricle. The catheter in the left atrium was introduced with the described transeptal technique.

tube may then be withdrawn, so that only the soft teflon catheter is left behind. By introducing a spiral metal guide into the catheter one makes the latter sufficiently stiff for further attempts at catheterization of the left ventricle with fluoroscopy. Fig. 3 shows how the catheter with inserted metal guide has been introduced into the left ventricle. If one does not succeed in pushing this catheter into the left ventricle, further attempts to obtain left ventricular pressure are made by introducing into the teflon catheter a polyethylene catheter (size PE 50) or nylon catheter. With the latter procedure there is of course the possibility of performing simultaneous pressure measurements from the left atrium and ventricle.

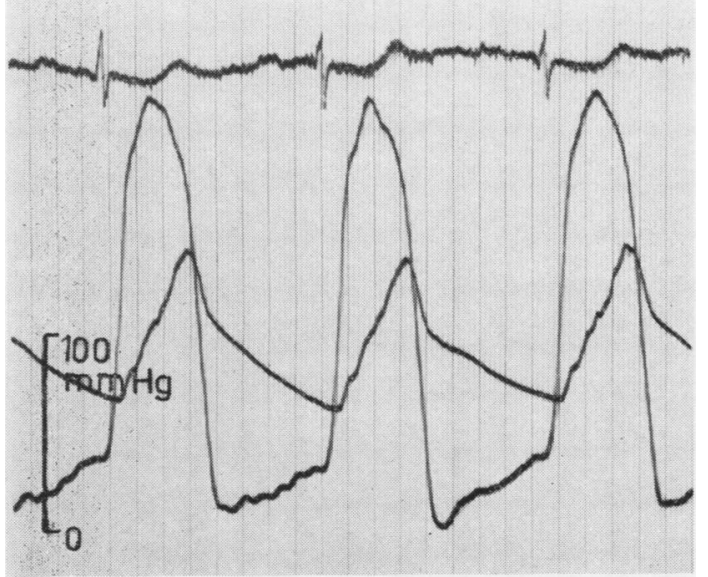

FIG. 5.-Simultaneous pressure recordings over the aortic valve in a case of severe aortic stenosis.

\section{RESULTS}

The instrument and the technique were first thoroughly tried out on 13 cadavers. In 12 of these it was easy to push the instrument down the vein. One felt immediately when the tip of the instrument glided down from the superior vena cava to the right atrium, and it was possible to palpate the septum. Several blind punctures performed in this way all proved on subsequent examination to have gone through the atrial septum quite near the site of the foramen ovale.

Seventeen patients with valve defects in the left side of the heart have been examined with this method. In three cases in which it was intended to carry out this examination, however, it was not possible to introduce the instrument, probably on account of unfavourable anatomical conditions. Of the 17 cases in which it was possible to introduce the instrument, pressure from the left atrium was obtained in 15 cases and pressure also from the left ventricle in nine cases. In one, pressure from the left ventricle was obtained directly in connexion with the puncture. In four, pressures in the left heart and cardiac output were determined also during leg exercise. In six cases the oxygen uptake and pulse rate were estimated before and after the introduction of the catheter into the left side of the heart. Left heart catheterization did not appreciably change the basal conditions.

The teflon catheter has good properties for the reproduction of pressures. Fig. 4 shows simultaneous registration of pressure from the left 
atrium and ventricle in a patient who had combined mitral disease. Pressure recordings from the left ventricle and aorta in a case with aortic stenosis examined with this method are shown in Fig. 5.

No serious complications have occurred. In two of the cases in which the attempt at puncture had to be repeated before pressure from the left atrium was obtained, it is probable that the pericardium was punctured. The patients were unaffected, but for a day or so had slight substernal pains and transient E.C.G. changes of pericarditis type. One of these was operated upon some days later for mitral stenosis, and a slight amount of blood was found in the pericardial fluid.

\section{Discussion}

As shown by Morrow et al. (1960), transeptal catheterization of the left atrium can be performed without serious complications. To enable leg exercise during the left heart catheterization we have used the transeptal puncture technique through the right external jugular vein.

The ability to pass the catheter from the left atrium to the ventricle is dependent on the anatomical conditions. In the case of a marked mitral stenosis combined with a sharp regurgitant jet and a very large left atrium, it may obviously be difficult to pass a thin polyethylene tube through the orifice. The chance might be better with the stiffer teflon catheter moved under the control of screening. Passing the catheter from the superior vena cava to the right atrium the direction is straight against the mitral orifice.

The procedure is not harmful for the patient. The metal tube may occasionally cause some pain in the region of the right shoulder. After removal of the metal tube, when only the catheter remains in the vein, this discomfort disappears. The catheter can remain within the left side of the heart for the time needed to get the patient into a steady $\frac{\bar{\sigma}}{\bar{\omega}}$ state for estimation of cardiac output and pressures $\vec{\nabla}$ both at rest and during exercise. The "basic" $\varrho$ condition of the patient was not disturbed by the procedure. There is no risk of air embolism from $\overrightarrow{0}$ the incision in the jugular vein, as the head is not $\overrightarrow{.}$ elevated and the veins are distended with blood $\vec{\omega}$ when the instrument is introduced.

\section{SUMMARY}

A method for transeptal catheterization of the left side of the heart with an instrument pushed 0 down from the external jugular vein has been described. It has been applied in 17 cases with valvular defects in the left heart. In 15 cases $\vec{\nabla}$ pressure from the left atrium was obtained, and $\Phi$ in nine cases pressure from the left ventricle also. $\stackrel{\mathbb{S}}{\stackrel{1}{2}}$ The method permits haemodynamic studies of the left side of the heart both at rest and during $\stackrel{\mathbb{C}}{-}$ exercise. No complications of a serious nature $\overrightarrow{0}$ were observed.

This investigation has been supported by a grant from A. B. Stille-Werner, Stockholm.

\section{REFERENCES}

Allison, P. R., and Linden, R. J. (1953). Circulation, T, 669. Bagger, M., Björk, V. O., and Malmström, G. (1957). Amer. Heart J. $53,91$. Björk, V. O., Malmström, G., and Uggla, L. G. (1953). Ann. Surg. $\frac{\circ}{3}$

Brock, R., Milstein, B. B., and Ross, D. N. (1956). Thorax, 11, 163ح Facquet, J., Lemoine, J. M., Alhomme, P., and Lefebvre, J. (1952)

Arch. Mal. Coeur, 45, 741.
Kjellberg, S. R., Nordenström, B., Rudhe, U., Björk, V. O., and Malmström, G. (1960). Acta radiol. (Stockh.), Suppl.

Morrow, A. G., Braunwald, E., and Ross, J. (1960). A.M.A. Arch? intern. Med., 105, 645 .

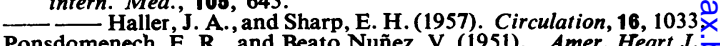
41, 643 .

Radner, S. (1955). Acta med. scand., 151, 223.

Radner, S. (1955). Acta med. scand.,

Ross, J. (1959). Ann. Surg., 149, 395.
Swan, H. J. C. (1959). Personal communication.

Zimmerman, H. A., Scott, R. W., and Becker, O. N. (1950)马 Circulation, 1, 357 . 